\title{
Electrostatic interaction of a pointlike charge with a wormhole
}

\author{
S. Krasnikov
}

\begin{abstract}
A pointlike electric charge at rest is considered in the spacetime which is a wormhole connecting two otherwise Minkowskian spaces. The potential (i. e. a solution of the Maxwell equations) is split into two parts: one of them depends only on the value and location of the charge, while the other, on the contrary, does not change when the charge is quasistatically moved. Correspondingly, the former is interpreted as being generated by the charge, and the latter as being source-free. It is shown that all source-free potentials differ in the flux through the throat (no "multipoles without multipoles"), which enables one to interpret the flux as the "charge" of the wormhole in agreement with Wheeler's concept of "charge without charge". The potential generated by the charge differs from Coulomb's and thus a force (called "self-force") acts on the charge even in the absence of the source-free field. This force is found explicitly in the limit of vanishing throat length. The result differs from that obtained recently by Khusnutdinov and Bakhmatov.
\end{abstract}

\section{Introduction}

What electric force (if any) acts on a pointlike charge at rest outside a wormhole, if there are no more charges in the space? This question is of interest by, at least, two reasons. The first is its relation to the famous concept of "charge without charge" [1]. Suppose, in a flat region of a spacetime we observe, for all sufficiently large values of the radial coordinate $r$, the electric field

$$
\boldsymbol{E}=\frac{Q}{r^{3}} \boldsymbol{r}, \quad r>r_{0}
$$




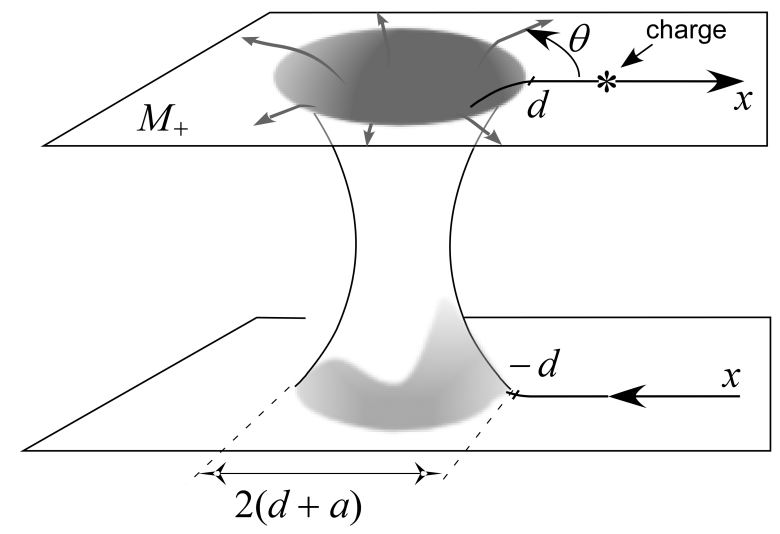

Figure 1: A section $\varphi=$ const of $M^{(3)}$. The divergent gray lines are the force lines of a source-free field which "imitates" the Coulomb field for an observer outside the sphere $r=d+a$.

From that we need not conclude that the field is generated by a charge (sitting, say, at at $r=0$ ). It well may happen that there is a wormhole mouth inside the sphere $r=r_{0}$ (so that the "coordinate" $r$ does not, in fact, take the zero value) and the field force lines do not terminate at all, see figure 1 . Which suggests that maybe there is no charge - as "substance" - in nature and the electromagnetic field is described by the source-free Maxwell equations, while all elementary "charges" are, in fact, mouths of wormholes? In developing such a theory it would be important to know how strong the resemblance is between a wormhole threaded by force lines and a pointlike charge $Q$. The flux conservation together with the spherical symmetry guarantees that the formula (1) for $\boldsymbol{E}$ is valid in both cases. So, one might think that, as long as we restrict ourselves to the region $r>r_{0}$, the resemblance is perfect. However, this is not the case: the force $\boldsymbol{F}$ experienced by a finite charge $q$ put in a point $p_{*}$ near the wormhole would not be just the Coulomb force $\boldsymbol{F}_{\mathrm{C}}=\frac{q Q}{r^{3}\left(p_{*}\right)} \boldsymbol{r}\left(p_{*}\right)$. And it is, in fact, the difference $\boldsymbol{F}_{\mathrm{s}}=\boldsymbol{F}-\boldsymbol{F}_{\mathrm{C}}$ that is the subject of this paper. As we shall see, not only $\boldsymbol{F}_{\mathrm{s}} \neq 0$, but $\boldsymbol{F}_{\mathrm{s}}$ can even overpower $\boldsymbol{F}_{\mathrm{C}}$.

Another problem to which the self-force is relevant is the possible existence of macroscopic traversable wormholes. At the moment the only ob-

\footnotetext{
${ }^{1}$ Throughout the paper we regard the matter supporting the wormhole as electrically neutral.
} 
servational restriction on their abundance has been obtained on the basis of unusual lensing properties of negative mass [2] and is valid only for a very special type of wormholes. To improve the situation it would be desirable, of course, to know more about physical effects involving wormholes. The consideration of the electrostatic problem in the wormhole background can be viewed as a step in that direction. An interesting, in this sense, result of this paper is that self-interaction leads to appearance of the attraction infinitely growing (in the approximation of infinitely short throat) as the charge approaches a mouth of a wormhole. Tempted by the resemblance between the electrostatic and (Newton's) gravitational forces — which differ in the sign, though — one might speculate therefore that wormholes are possible which are macroscopic and static, but nevertheless non-traversable for massive bodies.

Our analysis will be confined to a simplest wormhole:

$$
\begin{array}{ll} 
& \mathrm{d} s^{2}=-\mathrm{d} t^{2}+\mathrm{d} x^{2}+r^{2}(x)\left(\mathrm{d} \theta^{2}+\sin ^{2} \theta \mathrm{d} \varphi^{2}\right) \\
x \in \mathbb{R}, \quad r \in C^{\infty}, \quad r(-x)=r(x), \quad r>0,\left.\quad r\right|_{x>d}=x+a .
\end{array}
$$

The wormhole is obviously static and spherically symmetric. Each its spacelike section $t=$ const - we shall denote such sections by $M^{(3)}$ — is a pair of flat three-dimensional spaces $M_{+}$and $M_{-}$(they are defined by the inequalities $x>d$ and $x<-d$, respectively, and either is just the Euclidean space minus a ball of radius $d+a$ ) connected with a 'tunnel', see figure 1 .

The electric force experienced by a charge $q$ in $p_{*} \in M_{+}$is

$$
\boldsymbol{F}=-q \nabla \Phi
$$

where $\Phi$ is a solution of the (three-dimensional) equation

$$
\Phi_{, a}{ }^{; a}(p)=-4 \pi q \delta\left(p-p_{*}\right), \quad p \in M^{(3)} .
$$

Note. Equation (4a) - in which $a=1,2,3$ and the covariant derivative is understood as acting in the three-dimensional $M_{+}$- follows from the Maxwell equations written for the vector-potential $A^{i}, i=0 \ldots 3$. As is well known [3, problem 14.16], in curved spacetime those equations have, in the general case, two non-equivalent versions . Fortunately, $R_{i}^{0}=0$ for our metric and the difference does not lead to any ambiguity in the equation on $\Phi=A^{0}$.

There are two problems in finding the force $\boldsymbol{F}$ via eqs. (3), (4a). The minor one is that $\Phi$ diverges in $p_{*}$, where the force is to be found, and thus 
one needs a "renormalization" procedure to derive a meaningful and finite value for the force. The problem is quite hard in the general case (some relevant references can be found in [4]), but in the case under discussion $p_{*}$ is restrict to $M_{+}$, where the procedure is trivial due to flatness. A more serious problem is that eq. (4a) has too many solutions: if some $\Phi_{1}$ solves (4a) than so also does $\Phi_{1}+Z$, where $Z$ is "a source-free" potential, i. e. an arbitrary harmonic function. Correspondingly, the question arises as to which of those solutions should be interpreted as "the potential generated by the charge". In the ordinary electrostatics the problem is solved by requiring the electric field to fall at infinity

$$
\Phi,_{i} \rightarrow 0 \quad \text { at } r \rightarrow \infty
$$

which physically means that we are not interested in field configurations with infinite energies. We adopt the restriction (4b) too, but in our case this does not fix the problem, because in $M^{(3)}$ there are non-zero harmonic functions satisfying (4b). Thus, a pointlike charge near the wormhole can experience a host of forces of equal physical and mathematical status. Which means that to find the self-force acting on a charge one needs i) a solution of (4) and ii) a prescription for splitting it into two terms - the self-force potential $\Phi_{\mathrm{S}}$ and the source-free potential $\Phi_{\mathrm{s}-\mathrm{f}}$. What prescription to use is, of course, a matter of convention, but to be consistent one obviously should secure the independence of $\Phi_{\text {s-f }}$ from the location of the charge. In other words, if the charge is moved from $p_{*}$ to some other point $p_{* *}$ (it is understood that the charge is transported quasistatically, so that the radiation can be neglected) one would expect $\Phi_{\text {s-f }}$ to remain unchanged (otherwise why call it source-free). To summarize, the notion of self-force makes sense (only) if one can present a solution of (4) as a sum $\Phi=\Phi_{\mathrm{s}}+\Phi_{\mathrm{s}-\mathrm{f}}$, where the first term is defined uniuely by $p_{*}$ and $q$, while the second does not change at (quasistatical) motions of the charge. But the existence of such splitting looks unlikely in the general case.

Fortunately, in the case in question the problem admits a reasonable solution. As follows from the proposition proved in the next section, in the space $M^{(3)}$ all bounded harmonic functions are proportional (up to an irrelevant constant) to a single function $(\rho / r$ in the notation of section 2$)$. In $M_{+}$every such function has the form $Q / r+\Phi_{0}$ and the flux of its gradient through the sphere $x=d$ is obviously proportional to $Q$. Which allows us to uniquely define the self-force potential $\Phi_{\mathrm{s}}$ as the (renormalized) solution of eq. (4) with the zero flux of the gradient through the sphere $x=d$. The 
force acting on a charge $q$ in $p_{*} \in M_{+}$is then simply $-q \nabla \Phi_{\mathrm{S}}-\frac{q Q}{r^{3}} \boldsymbol{r}$. Due to the Maxwell equations $Q$ is conserved, so the second term does not change when the charge moves and thus can be considered source-free.

Note. Recently, Khusnutdinov and Bakhmatov [4] have found special solutions - let us call them $\Phi_{\mathrm{KB}}^{(1)}$ and $\Phi_{\mathrm{KB}}^{(2)}$ — of eqs (4) for $r=a+|x|$ and $r=\sqrt{a^{2}+x^{2}}$, respectively (later $\Phi_{\mathrm{KB}}^{(2)}$ was reproduced by Linet [5], who used a different method). Neither of those $r(x)$ satisfies (2b), but it seems, nevertheless, very plausible that our approach is valid for them too. If so, the mentioned solutions correspond not to the self-interaction of the charge (the flux of $\nabla \Phi_{\mathrm{KB}}^{(1)}$ through the sphere $x=$ const is non-zero), but rather to the sum of its self-interaction and interaction with the wormhole of non-zero charge $Q_{\mathrm{KB}}$. It is crucial that $Q_{\mathrm{KB}}$ depends on $p_{*}$ (in particular, the comparison of our formula (17) with that for $G^{r e n}$ in [4] gives for the wormhole of the first type $\left.Q_{\mathrm{KB}}=-\frac{q a}{2 r_{*}}\right)$. So, if, following [4], one choose to interpret $\Phi_{\mathrm{KB}}^{(1)}$ as the potential generated by the charge in $p_{*}$, one would have to admit that moving the charge one manages somehow to change at the same time the source-free field too.

\section{The multipole expansion}

In this section we establish the uniqueness of the solution of equation (4) up to the term $Q \rho / r+\Phi_{0}$, where $\rho$ is a certain function (specified below), while $Q$ and $\Phi_{0}$ are arbitrary constants .

We begin with rewriting equation (4a) in the coordinate form

$$
\left[\partial_{x}^{2}+\frac{2 r^{\prime}}{r} \partial_{x}+\frac{1}{r^{2}}\left(\partial_{\theta}^{2}+\cot \theta \partial_{\theta}+\sin ^{-2} \theta \partial_{\varphi}^{2}\right)\right] \Phi=-\frac{4 \pi q}{r^{2} \sin \theta} \delta(\varphi) \delta(\theta) \delta\left(x-x_{*}\right)
$$

(we have set $\varphi_{*}=\theta_{*}=0$, which obviously does not lead to any loss of generality). Expanding

$$
\Phi=\sum_{l=0}^{\infty} \sum_{m=-l}^{l} \phi_{l}^{(m)}(x) Y_{l}^{m}(\varphi, \theta),
$$

where $Y_{l}^{m}$ are spherical functions [6]

$$
Y_{l}^{m}(\varphi, \theta) \equiv \sqrt{\frac{2 l+1}{4 \pi} \frac{(l-|m|) !}{(l+|m|) !}} \mathcal{P}_{l}^{|m|}(\cos \theta) e^{i m \varphi},
$$




$$
\mathcal{P}_{l}^{m}(\cos \theta)(\mu) \equiv\left(1-\mu^{2}\right)^{\frac{m}{2}} \frac{\mathrm{d}^{m}}{\mathrm{~d} \mu^{m}} \mathcal{P}_{l}(\mu)
$$

$\left(\mathcal{P}_{l}\right.$ are the Legendre polynomials) one gets

$$
\begin{aligned}
\sum_{l=0}^{\infty} \sum_{m=-l}^{l}\left[\partial_{x}^{2}+\frac{2 r^{\prime}}{r} \partial_{x}-\frac{l(l+1)}{r^{2}}\right] \phi_{l}^{(m)}(x) & Y_{l}^{m}(\varphi, \theta) \\
& =-\frac{4 \pi q}{r^{2} \sin \theta} \delta(\varphi) \delta(\theta) \delta\left(x-x_{*}\right)
\end{aligned}
$$

Multiply both sides of (5) by $r Y_{l^{\prime}}^{m^{\prime} *} \sin \theta$ and integrate over $\varphi$ and $\theta$. The result $\left(Y_{l^{\prime}}^{m^{\prime}}\right.$ are ortonormal on the sphere) is

$$
\left[\partial_{x}^{2}-\left(\frac{r^{\prime \prime}}{r}+\frac{l(l+1)}{r^{2}}\right)\right] r \phi_{l}^{(m)}(x)=-\frac{4 \pi q}{r} \delta\left(x-x_{*}\right) Y_{l}^{m *}(0,0),
$$

It is convenient to treat the cases of zero and non-zero $m$ separately, because

$$
Y_{l}^{0}(0,0)=\sqrt{\frac{2 l+1}{4 \pi}}, \quad Y_{l}^{m}(0,0)=0, \quad m \neq 0
$$

So, we define

$$
v_{l} \equiv \sqrt{\frac{2 l+1}{4 \pi}} r \phi_{l}^{(0)}, \quad v_{l, m} \equiv r \phi_{l}^{(m)}, \quad m \neq 0 .
$$

For $v_{l}$ we have

$$
\left[\partial_{x}^{2}-\left(\frac{r^{\prime \prime}}{r}+\frac{l(l+1)}{r^{2}}\right)\right] v_{l}=-\frac{2 l+1}{r_{*}} q \delta\left(x-x_{*}\right)
$$

while $v_{l, m}$ irrespective of $m$ must solve the equation

$$
\left[\partial_{x}^{2}-\left(\frac{r^{\prime \prime}}{r}+\frac{l(l+1)}{r^{2}}\right)\right] z(l, x)=0 .
$$

Thus, the solution of eq. (4) is the function

$$
\Phi=\frac{1}{r} \sum_{l=0}^{\infty} v_{l}(x) \mathcal{P}_{l}(\cos \theta)+\frac{1}{r} \sum_{l=1}^{\infty} \sum_{|m|=1}^{l} v_{l, m}(x) Y_{l}^{m}(\varphi, \theta),
$$

where $v_{l}$ and $v_{l, m}$ are the solutions, respectively, of eqs. (6) and (7) which [because of (4b)] grow at $|x| \rightarrow \infty$ not faster than $|x|$.

To proceed note that in the flat regions $M_{ \pm}$the term with $r^{\prime \prime}$ vanishes in eq. (7) and the equation is easily solved: the solution is a superposition of $r^{-l}$ and $r^{l+1}$. 
Notation By $z_{+}$and $z_{-}$we denote the solutions of eq. (7) which are equalal to $r^{-l}$ at, respectively, $x>d$ and $x<-d$. And $z_{e}, z_{o}$ are the solutions of eq. (7) defined by the initial data

$$
z_{e}(0)=1, \quad z_{e}^{\prime}(0)=0, \quad z_{o}(0)=0, \quad z_{o}^{\prime}(0)=1
$$

Evidently $z_{e}$ and $z_{o}$ are even and odd, respectively, and any solution of eq. (7) is their linear combination.

Proposition. If $z(l, x)$ is a solution of eq. (7) with $l>0$, the function $r^{-1} z$ grows unboundedly as $x \rightarrow(-) \infty$.

Proof. First, let us rewrite eq. (7) as

$$
W^{\prime}(z, r)=\frac{l(l+1)}{r} z,
$$

where $W$ is the Wronskian $W\left(f_{1}, f_{2}\right)=f_{1}^{\prime} f_{2}-f_{1} f_{2}^{\prime}$. Integrating (9) we obtain

$$
\frac{z^{\prime}}{z}-\frac{r^{\prime}}{r}=\frac{1}{r z}\left[z^{\prime}\left(x_{0}\right) r\left(x_{0}\right)-z\left(x_{0}\right) r^{\prime}\left(x_{0}\right)\right]+\frac{l(l+1)}{r z} \int_{x_{0}}^{x} \frac{z \mathrm{~d} x}{r} .
$$

Now substitute $z=z_{e}$ and $z=z_{o}$ in that formula. Choose $x_{0}$ so as to make the first term in the right hand side non-negative, e. g., $x_{0}$ might be zero in the former case and a (sufficiently) small positive number in the latter. Then the r. h. s. is positive at least up to $x_{1}$, where $x_{1}$ is $\infty$, if $z(x)$ has no zeroes, and the first zero of $z$ otherwise. Thus, $z_{e(o)}(x) / r(x)$ grows at $x \in\left(x_{0}, x_{1}\right)$. But $r$ does not vanish. So, it follows that neither does $z_{e(o)}(x)$. Hence $x_{1}=\infty$ and therefore $z_{e(o)}(x) / r(x)$ grows at all $x>x_{0}$, including $x>d$. Which means that $z_{e(o)}(x)$ cannot be proportional to $r^{-l}$ at large $x$. Consequently,

$$
z_{e(o)}(x) \sim c_{2} r^{l+1}, \quad x \rightarrow \infty, \quad c_{2} \neq 0 .
$$

The same is true for $x \rightarrow-\infty$, since $z_{e(o)}$ is even (odd). And, finally, it is true, when $x \rightarrow \infty$ or $x \rightarrow-\infty$, for every $z$ because any of them is a superposition of $z=z_{e}$ and $z=z_{o}$.

Corollary 1. If $l>0$, the solutions $z_{+}$and $z_{-}$are linearly independent.

Corollary 2. The second term in the r. h. s. of (8) is zero. 
Corollary 3. Denote by $\vartheta$ the Heaviside step function. Then at $l>0$ the function

$$
v_{l}=-\frac{(2 l+1) q}{r_{*}} \frac{\vartheta\left(x-x_{*}\right) z_{+}(l, x) z_{-}\left(l, x_{*}\right)+\vartheta\left(x_{*}-x\right) z_{-}(l, x) z_{+}\left(l, x_{*}\right)}{W\left(z_{+}, z_{-}\right)}
$$

is the unique solution of eq. (6) that grows slower than $r^{l+1}$ at the infinities.

Now let us turn to the case $l=0$. Eq. (7) [as seen from (9)] transforms into $W^{\prime}(z, r)=0$. Which gives

$$
(z / r)^{\prime}=C / r^{2}
$$

where $C$ is an arbitrary constant. Thus, $z(0, x)$ is a linear combination of $r$ and $\rho$

$$
\rho \equiv \frac{r}{r(d)}-r \int_{d}^{x} \frac{\mathrm{d} x}{r^{2}(x)} .
$$

In this case $z_{+}$is proportional to $z_{-}$and the formula (10a) does not define $v_{0}$. The latter, however, can be easily found by using - as independent solutions of the homogeneous equation (7) — the functions $\rho$ and $r$ instead of $z_{+}$and $z_{-}($note that $W(r, \rho)=1)$ :

$$
v_{0}=\frac{q}{r_{*}}\left(\vartheta\left(x-x_{*}\right) \rho(x) r_{*}+\vartheta\left(x_{*}-x\right) r \rho_{*}\right)+Q \rho+\Phi_{0} r
$$

Here $\rho_{*}=\rho\left(x_{*}\right), r_{*}=r\left(x_{*}\right)$, and $Q, \Phi_{0}$ are arbitrary constants.

Summing up,

$$
\Phi=\frac{1}{r} \sum_{l=0}^{\infty} v_{l}(x) \mathcal{P}_{l}(\cos \theta),
$$

where $v_{l}(x)$ are given by formulas $(10)$.

\section{Self-interaction}

Eq. (11) gives, in principle, the electrostatic field of a pointlike charge in the wormhole background. However, to find the force acting on the charge it remains to cope with the fact that the field diverges in the point $p_{*}$ where the charge is located. To this end we take advantage of the fact that $p_{*}$ is in 
a flat part of the wormhole (let it be $M_{+}$, for definiteness). In this region we define the potential (for the second equality see, e. g., [6, eq. (II 2.13)])

$$
\begin{aligned}
\Phi_{\mathrm{Eucl}}(p) \equiv & \frac{q}{\left|p, p_{*}\right|}= \\
& \frac{1}{r} \sum_{l=0}^{\infty} q\left[\vartheta\left(x-x_{*}\right)\left(r_{*} / r\right)^{l}+\vartheta\left(x_{*}-x\right)\left(r / r_{*}\right)^{l+1}\right] \mathcal{P}_{l}(\cos \theta),
\end{aligned}
$$

where $\left|p, p_{*}\right|$ is the distance between $p$ and $p_{*}$ in the space $\mathbb{E}^{3}$, obtained by gluing a usual Eucleian ball of radius $d$ to $M_{+}$. From the usual electrostatics we know that the field $-\nabla \Phi_{\text {Eucl }}$ exerts no force on the charge. So, in finding the self-forcewe are only interested in the difference

$$
\Phi_{\text {ext }} \equiv \Phi-\Phi_{\text {Eucl }}
$$

(which is defined, of course, only in $M_{+}$). It is $\Phi_{\text {ext }}$ that plays the rôle of the "external field", i. e. the force acting on the charge is $\boldsymbol{F}=-q \nabla \Phi_{\text {ext }}\left(p_{*}\right)$.

To rewrite the expression (11) for $\Phi$ in a more convenient form let us substitute the equalities (in fact, the second one is a definition of $\alpha_{l}$ )

$$
z_{+}(x, l)=r^{-l}, \quad z_{-}(x, l)=C\left(r^{l+1}+\alpha_{l} r^{-l}\right), \quad \text { at } \quad l>0, x>d,
$$

into 10a):

$v_{l}=q\left[\vartheta\left(x-x_{*}\right)\left(r_{*} / r\right)^{l}+\vartheta\left(x_{*}-x\right)\left(r / r_{*}\right)^{l+1}+\frac{\alpha_{l}}{r_{*}}\left(r r_{*}\right)^{-l}\right], \quad$ at $\quad l>0, x, x_{*}>d$

Substituting this together with an obvious equality

$$
v_{0}=q\left[\vartheta\left(x-x_{*}\right)+\left(r / r_{*}\right) \vartheta\left(x_{*}-x\right)\right]+Q+r \Phi_{0}, \quad \text { at } \quad l>0, x, x_{*}>d .
$$

into (11) and, then, the result — combined with $(12)$ — into 113$)$, we finally obtain

$$
\Phi_{\mathrm{ext}}(p)=\Phi_{\mathrm{s}}(p)+\Phi_{\mathrm{s}-\mathrm{f}}(p), \quad p, p_{*} \in M_{+},
$$

where

$$
\Phi_{\mathrm{s}} \equiv q \sum_{l=1}^{\infty} \alpha_{l}\left(r r_{*}\right)^{-l-1} \mathcal{P}_{l}(\cos \theta), \quad \Phi_{\mathrm{s}-\mathrm{f}} \equiv Q / r+\Phi_{0} .
$$

We interpret $\Phi_{\mathrm{s}}$ and $\Phi_{\mathrm{s}-\mathrm{f}}$ as the parts of $\Phi_{\text {ext }}$ generated by the charge and by the wormhole, respectively. To justify that interpretation note that 1) $\Phi_{\mathrm{s}}$, 
for a given $p$, depends only on $q$ and $p_{*}$ and 2) $\Phi_{\text {s-f }}$, on the contrary, does not depend on $p_{*}$ in the following sense. Suppose the charge is at rest up to some moment $t_{0}$ and is then quasistatically moved from $p_{*}\left(t_{0}\right)$ to some $p_{*}\left(t_{1}\right) \in M_{+}$, where - at the moment $t_{1}$ - is again put to rest. Let $t_{2}$ be a moment when at small $|x|$ the disturbance in the potential caused by the motion of the charge has already settled down and the potential became constant (in time) $)^{2}$. Then at times $t>t_{2}$ in the vicinity of the wormhole the equations (15) remain valid with $r_{*}\left(t_{0}\right)$ replaced by $r_{*}\left(t_{1}\right)$ and with the same $Q$.

Proof. Indeed, at $t>t_{2}$ the flux of $\nabla \Phi$ through the sphere $x=d$ is $\mathcal{F}\left(d, t_{2}\right)=$ $-4 \pi Q\left(t_{2}\right)$, because neither $\Phi_{\text {Eucl }}$, nor $\Phi_{\mathrm{s}}$ give any contribution to it. At the same time, there is a sphere $x=D>x_{*}$ such that $\mathcal{F}\left(D, t_{2}\right)=-4 \pi\left[Q\left(t_{0}\right)+q\right]$, because if $D>x_{*}+c\left(t-t_{0}\right)$, the field is not disturbed there yet. Thus the flux $\mathcal{F}_{B}\left(t_{2}\right)$ through the boundary of the layer $\left\{d<x<D, t=t_{2}\right\}$ is $4 \pi\left[Q\left(t_{2}\right)-Q\left(t_{0}\right)-q\right]$. On the other hand, the total charge inside the layer has not changed and hence $\mathcal{F}_{B}\left(t_{2}\right)=\mathcal{F}_{B}\left(t_{0}\right)=-4 \pi q$ by the Gauss theorem. So, $Q\left(t_{2}\right)=Q\left(t_{0}\right)$.

\section{Short wormhole}

It is seen from formulas $(15)$ that the force acting on a charge depends on the form of the wormhole - the information about the form being encoded in the coefficients $\alpha_{l}$. But today we have no reasons to consider any particular form as more realistic than any other. So, it would be interesting to find a form-independent effect. To this end we consider in this section the limit $d \rightarrow 0$ for the wormhole (2) with $a>0$. In doing so we allow the the throat to be arbitrary, the only additional requirement on $r(x)$ being

$$
r^{\prime}<c_{r} \quad \forall d
$$

$\left(c_{r}\right.$ is a constant), which, among other things, guarantees that $r(0) \rightarrow a$.

\footnotetext{
${ }^{2}$ The existence of such a moment is an assumption, even though a very plausible one. If a wormhole is such that in its vicinity the electro-magnetic waves caused by stirring the charge do not dissipate with time, one probably cannot develop electrostatics in that spacetime at all.
} 
Let us, first, present $z_{-}$as the solution of the following differential equation (which does not contain the large quantity $r^{\prime \prime}$ )

$$
\begin{gathered}
{\left[\partial_{x}^{2}+2 r^{\prime} r^{-1} \partial_{x}-l(l+1) r^{-2}\right] y=0,} \\
y(-d)=(d+a)^{-l-1} .
\end{gathered}
$$

Here the first line is simply eq. (7) in terms of $y \equiv z_{-} / r$, while the second follows from the definition of $z_{-}$. The coefficients of the equation by (16) are uniformly (by $d$ ) bounded, so at a fixed $l$ and $d \rightarrow 0$

$$
\ln ^{\prime} y(-d) \rightarrow \ln ^{\prime} y(d) .
$$

Hence, $\ln ^{\prime} z(-d)-\ln ^{\prime} r(-d) \rightarrow \ln ^{\prime} z(d)-\ln ^{\prime} r(d)$ and thus

$$
\ln ^{\prime} z_{-}(d) \rightarrow \ln ^{\prime} z_{-}(-d)+2 / a \rightarrow(l+2) / a
$$

(recall that $z_{-}=(a-x)^{-l}$ at $\left.x=-d\right)$. On the other hand, by (14)

$$
\ln ^{\prime} z_{-}(d)=\left.\frac{(l+1) r^{l}-l \alpha_{l} r^{-l-1}}{r^{l+1}+\alpha_{l} r^{-l}}\right|_{r=a}=\frac{(l+1) a^{l}-l \alpha_{l} a^{-l-1}}{a^{l+1}+\alpha_{l} a^{-l}},
$$

combining which with the equation above we find in the limit $d \rightarrow 0$

$$
\alpha_{l}=-\frac{a^{2 l+1}}{2(l+1)} .
$$

Thus, asymptotically,

$$
\begin{aligned}
\Phi_{\text {ext }} \sim-q \sum_{l=1}^{\infty} \frac{a^{2 l+1}}{2(l+1)}\left(r r_{*}\right)^{-l-1} & \mathcal{P}_{l}(\cos \theta)+\frac{Q}{r}+\Phi_{0}= \\
& -\frac{q}{2 a} \sum_{l=2}^{\infty} \frac{1}{l}\left(a^{2} / r r_{*}\right)^{l} \mathcal{P}_{l-1}(\cos \theta)+\frac{Q}{r}+\Phi_{0}
\end{aligned}
$$

In particular, at $\theta=0$

$$
\begin{aligned}
\Phi_{\text {ext }} \sim-\frac{q}{2 a} \sum_{l=2}^{\infty} \frac{1}{l}\left(a^{2} / r r_{*}\right)^{l}+\frac{Q}{r} & +\Phi_{0} \\
& =\frac{q}{2 a}\left[\ln \left(1-\frac{a^{2}}{r r_{*}}\right)+\frac{a^{2}}{r_{*} r}\right]+\frac{Q}{r}+\Phi_{0}
\end{aligned}
$$


and the electric field on the axis is

$$
-\Phi,_{x}=-\left(\Phi_{\text {ext }}+\Phi_{\text {Eucl }}\right)_{x} \sim q \frac{r-r_{*}}{\left|r-r_{*}\right|^{3}}+\frac{q a^{3}}{2 r_{*} r^{2}\left(a^{2}-r r_{*}\right)}+\frac{Q}{r^{2}} .
$$

Thus, asymptotically, in the presence of an infinitely short wormhole with the radius $a$ a pointlike charge $q$ experiences the (radial) force

$$
F\left(r_{*}\right)=-q \Phi_{\mathrm{ext}, x}=-\frac{q^{2} a^{3}}{2 r_{*}^{3}\left(r_{*}^{2}-a^{2}\right)}+\frac{q Q}{r_{*}^{2}} .
$$

Its first term — the self-force $\boldsymbol{F}_{\mathrm{s}}$ — can be presented, if desired, in the form

$$
\boldsymbol{F}_{\mathrm{S}}(r)=-\nabla U(r), \quad U(r)=\frac{q^{2}}{4 a}\left(\ln \left[1-(a / r)^{2}+(a / r)^{2}\right]\right)
$$

\section{Acknowledgements}

I am grateful to N. R. Khusnutdinov and R. R. Zapatrin for an inspiring discussion on the subject. This work was supported by RNP Grant No. 2.1.1.6826.

\section{References}

[1] J. A. Wheeler, Geometrodynamics, (Academic Press, New York, 1992).

[2] D. F. Torres, G. E. Romero, and L. A. Anchordoqui Phys. Rev. D 58 (1998) 123001.

[3] A. P. Lightman et al., Problem Book in Relativity and Gravitation, (Princeton University Press, Princeton, 1975).

[4] N. R. Khusnutdinov and I. V. Bakhmatov Phys. Rev. D 76 (2007) 124015 .

[5] B. Linet, Electrostatics in a wormhole geometry, e-print: arXiv:0712.0539.

[6] V. V. Batygin and I. N. Toptygin, Problems in Electrodynamics, (Academic Press Inc.,U.S., 1978). 